\title{
Gas6 expression is reduced in advanced breast cancers
}

\author{
Ayman M. Ibrahim ${ }^{1,2,5}$, Zane Gray ${ }^{1,5}$, Angelica M. Gomes ${ }^{1,5}$, Leann Myers $\mathbb{D}^{3}$, Fariba Behbod ${ }^{4}$ and Heather L. Machado ${ }^{1 凶}$
}

Growth arrest-specific gene 6 (Gas6) is a cytokine that binds to receptor tyrosine kinases Tyro3, Axl, and Mer. Numerous studies have suggested that macrophage-derived Gas6 interacts with AxI to promote cancer progression, and Axl has been associated with poor clinical outcome. However, the expression and relevance of Gas6 in human breast cancer patients has not been studied. Analysis of tissue microarrays showed that Gas6 was highly expressed in ductal carcinoma in situ (DCIS) but markedly decreased in invasive breast cancer. Gas6 and Axl were weakly correlated, suggesting that their functions may not exclusively rely on each other. Analyses of publicly available databases showed significantly improved overall and relapse-free survival in patients with high Gas6 mRNA, particularly in luminal A breast cancers. These findings indicate that tumor-derived Gas 6 is not overexpressed in invasive breast cancer, and may not be a negative prognostic factor in human breast cancer.

npj Precision Oncology (2020)4:9; https://doi.org/10.1038/s41698-020-0116-z

\section{INTRODUCTION}

Gas6 is a vitamin K-dependent cytokine that binds to a family of receptor tyrosine kinases that includes Tyro3, Axl, and Mer (TAMR family), with 100-1000 times higher affinity for Axl ${ }^{1,2}$. Gas6/TAMR signaling is a critical component of the innate immune response, functions in phagocytic clearance of apoptotic cells and is an important thrombosis factor. More recently, Gas6 was shown to modulate different cellular events such as proliferation, survival and invasion in vitro ${ }^{3-5}$. In vitro studies have suggested that Gas6/ Axl signaling promotes tumor cell survival and invasion, such as osteosarcoma $^{6}$, hepatocellular carcinoma ${ }^{7}$, renal cell carcinoma ${ }^{8}$, and lung cancer ${ }^{9}$. In breast cancer, Axl is highly expressed in triple negative breast cancer (TNBC) cell lines ${ }^{10}$ and is correlated with poor clinical outcome (reviewed in ref. ${ }^{11}$ ). In addition, studies using mouse models have shown that macrophage-derived Gas6 may have a tumor-promoting role in cancer progression ${ }^{12}$. In contrast, other studies revealed Gas6-independent functions of Axl including regulation of epithelial-mesenchymal-transition and breast cancer metastasis ${ }^{10,13,14}$. Axl can be activated by other RTKs, including EGFR and VEGFR2, and has been associated with resistance to targeted therapies in lung, pancreatic, and breast cancers $^{15-17}$.

In the past decade, there have been tremendous efforts in targeting Axl, and more recently Gas6, as a therapeutic strategy for metastatic breast cancer. However, few studies have addressed the expression of Gas 6 and relation to overall survival in human breast cancers. In this study, we assessed the expression pattern of Gas6 at different stages and subtypes of human breast cancer. To our surprise, tumor-derived Gas6 significantly decreased in breast cancer as compared with normal tissues, and overall survival and relapse-free survival (RFS) was significantly improved in breast cancers with high Gas6 expression. In support, Gas6 mRNA and protein expression was reduced in luminal $\mathrm{B}, \mathrm{Her} 2^{+}$, and TNBC, which may suggest that Gas 6 overexpression is not a negative prognostic factor.

\section{RESULTS}

Gas6 expression declines in invasive breast tumors

We previously reported that Gas6 was expressed in macrophages and pre-invasive epithelial cells of ductal carcinoma in situ $(D C I S)^{18}$, the non-obligatory precursor of invasive ductal carcinoma (IDC). To expand these studies, we stained two tissue microarrays (TMAs) (Table 1) with an antibody that recognizes human Gas6, and scored epithelial staining based on intensity and the number of stained cells. Antibody specificity was validated by staining NCl-H226 cells (positive control) or HT29 cells (negative control) (Supplementary Fig. 1a). For TMAs, epithelial Gas6 expression was scored on a scale from 0 to 3 and represented as negative (0), low (1), moderate (2), or high (3) (Fig. 1a). In support of our previous studies in the mouse mammary gland ${ }^{19}$, Gas6 was highly expressed in the ductal epithelium of normal breast (adjacent normal). Compared to normal tissue, there was a significant increase in Gas6 protein in pure DCIS. On the other hand, Gas6 expression was decreased in DCIS with adjacent IDC (DCIS + IDC), with the lowest expression in IDC alone (Fig. 1b, c, and Supplementary Fig. 1b). Notably, Gas6 staining was observed throughout the cytoplasm and concentrated near the ductal lumens in normal breast, whereas Gas6 appeared to be localized to specific cytoplasmic compartments in IDC. Stromal Gas6 expression was unchanged amongst groups (Supplementary Fig. 1c). We also analyzed Gas6 mRNA in the GEPIA breast cancer dataset, an online platform that employs data from the TCGA and GTex projects ${ }^{20}$. Interestingly, multiple adjacent normal female reproductive tissues including breast, uterus, ovary, and cervix expressed higher levels of Gas6 mRNA than their tumor counterparts (Supplementary Fig. 2a). Further analysis revealed a statistically significant reduction in Gas6 (RNA transcripts per million) in breast cancer $(n=1085)$ compared with normal $(n=$ 291) (Supplementary Fig. 2b). These data suggest that Gas6 mRNA is highly expressed in normal female reproductive tissues but decreased in invasive cancers.

\footnotetext{
${ }^{1}$ Department of Biochemistry and Molecular Biology, Tulane Cancer Center, Tulane School of Medicine, New Orleans, LA, USA. ${ }^{2}$ Department of Zoology, Faculty of Science, Cairo University, Giza, Egypt. ${ }^{3}$ Department of Biostatistics and Data Science, Tulane University School of Public Health and Tropical Medicine, New Orleans, LA, USA. ${ }^{4}$ Department of Pathology and Laboratory Medicine, University of Kansas Medical Center, Kansas City, KS, USA. ${ }^{5}$ These authors contributed equally: Ayman M. Ibrahim, Zane Gray, Angelica M. Gomes. ${ }^{{ }^{2}}$ email: hmachado@tulane.edu
} 
Table 1. Histopathological characteristics of patient cohort used in the IHC analysis.

\begin{tabular}{llll}
\hline Parameter & $\begin{array}{l}\text { Pure DCIS } \\
(n=12)(\%)\end{array}$ & $\begin{array}{l}\text { DCIS }+ \text { IDC } \\
(n=44)(\%)\end{array}$ & $\begin{array}{l}\text { IDC } \\
(n=100)(\%)\end{array}$ \\
\hline $\begin{array}{l}\text { Age (yrs) } \\
\text { Range }\end{array}$ & $36-81$ & $41-91$ & $29-95$ \\
Mean & $53(13.3)$ & $58.16(12.11)$ & $50.65(12.3)$ \\
Tumor grade & & & \\
$\begin{array}{l}\text { Grade I } \\
\text { Grade II }\end{array}$ & $8.3 \%$ & $0 \%$ & $6 \%$ \\
Grade III & $66.7 \%$ & $45.7 \%$ & $72.7 \%$ \\
ER status & $25 \%$ & $54.3 \%$ & $21.3 \%$ \\
$\begin{array}{l}\text { Positive } \\
\text { Negative }\end{array}$ & $9 \%$ & & \\
PR status & $91 \%$ & $73.8 \%$ & $68 \%$ \\
$\begin{array}{l}\text { Positive } \\
\text { Negative }\end{array}$ & $18.2 \%$ & $26.2 \%$ & $32 \%$ \\
Her2 status & $81.8 \%$ & $61.7 \%$ & $57 \%$ \\
$\begin{array}{l}\text { Positive } \\
\text { Negative }\end{array}$ & $36.4 \%$ & $39.3 \%$ & $43 \%$ \\
Ki67 & $63.6 \%$ & $23.23 \%$ & $40 \%$ \\
Low & $63.5 \%$ & $76.76 \%$ & $60 \%$ \\
High & $36.5 \%$ & $62.4 \%$ & $64 \%$ \\
\hline $\begin{array}{l}\text { The cohort included patients with DCIS, IDC, and with different molecular } \\
\text { subtypes. }\end{array}$ & & & \\
\hline
\end{tabular}
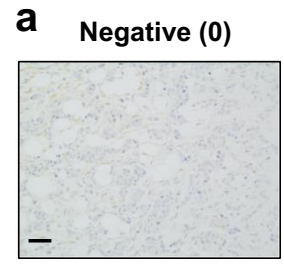

b
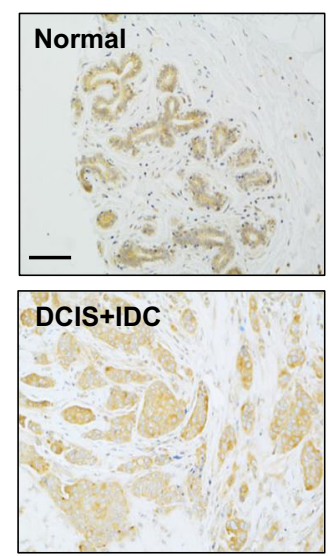

Low (1)

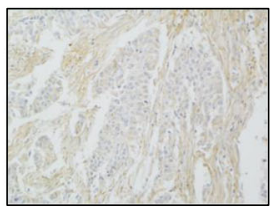

Gas6 expression is decreased in luminal $\mathrm{B}, \mathrm{Her}^{+}$, and basal-like breast cancers

To determine whether Gas6 is preferentially expressed in a particular breast cancer subtype, we categorized patient samples from the TMAs as luminal A, luminal $\mathrm{B}, \mathrm{Her}^{+}$and TNBC, based on clinical attributes including hormone receptor status, Her2 status, and Ki67 expression. The majority of patient samples expressed moderate levels of epithelial Gas6 regardless of subtype, although a subset of patients with TNBC did not express Gas6 (Fig. 2a). Interestingly, luminal A breast cancers, which generally have a favorable outcome, showed the largest percentage of patients with high Gas6 expression (Fig. 2a and Supplementary Fig. 3a). The localization and pattern of Gas6 staining was consistent among all breast cancer subtypes. Stromal protein expression of Gas6 was also quantified, and showed no distinct difference between subtypes, nonetheless, a small subset of luminal B and $\mathrm{Her}^{+}$patients had the highest Gas6 expression (Supplementary Fig. 3b)

Next, we used cBioportal ${ }^{21}$ to analyze breast cancer patients from the METABRIC $(n=1904)^{22}$ and TCGA $(n=481)^{23}$ data sets. Patients in each data set were grouped by PAM50 score, a tumor profiling test of 50 known genes in breast cancer, to plot Gas6 mRNA levels across distinct molecular subtypes. In METABRIC data, patients with luminal B or Her2 ${ }^{+}$expressed significantly lower levels of Gas6 mRNA relative to non-cancerous breast tissue (Fig. 2b). Similarly, analysis of TCGA data showed that patients with luminal $\mathrm{B}, \mathrm{Her}^{+}$, and basal-like (which includes TNBC) tumors expressed significantly lower levels of Gas6 mRNA, relative to normal-like cancers (Fig. 2c). These results suggest that Gas6 is expressed amongst all breast cancer subtypes but is lowest in breast cancers that are commonly characterized by poor outcome.

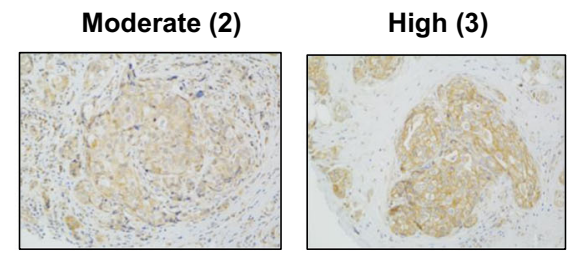

C
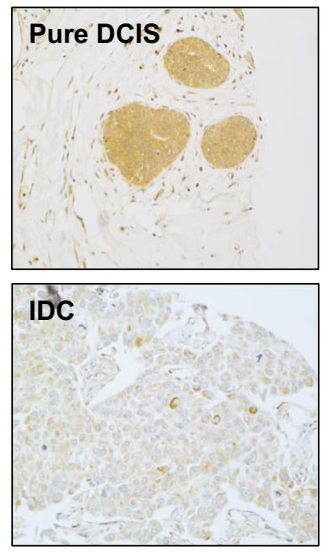

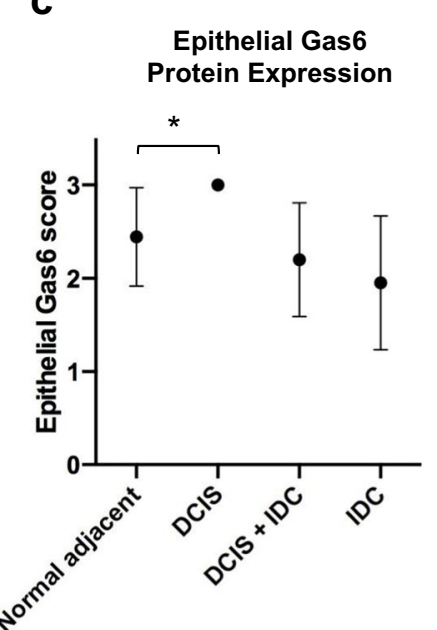

Fig. 1 Gas6 expression in normal breast and invasive breast cancer. a Representative images of epithelial Gas6 staining and the corresponding score assigned as negative (0), low (1), moderate (2), or high (3). b Immunohistochemical analysis of Gas6 in normal breast tissue and different stages of cancer, showing the localization and the differential expression of Gas6. c Graph depicts epithelial Gas6 score in the epithelium of normal breast and different stages of breast cancer (normal: $n=9$, pure DCIS: $n=12$, DCIS/IDC: $n=44$, IDC: $n=144$ ). Error bars are SEM, ${ }^{*} p=0.008$ (Chi-squared test). Scale bars $=100 \mu \mathrm{m}$. 
a

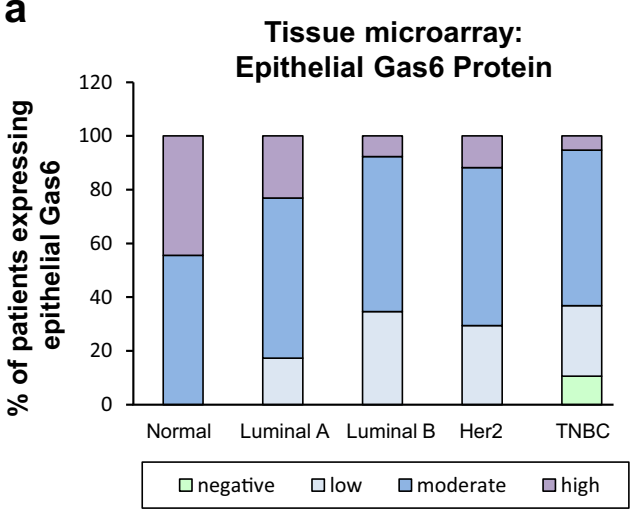

b

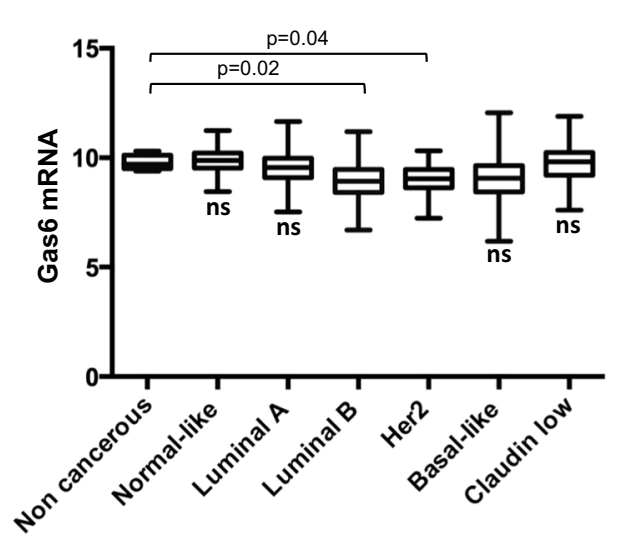

C

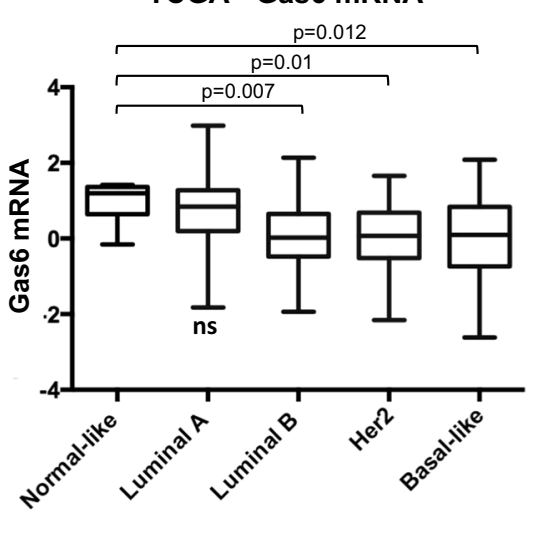

Fig. 2 Gas6 expression across breast cancer subtypes. a Graph depicts quantitation Gas6 protein staining of TMAs, expressed as the percentage (\%) of patients expressing epithelial Gas6 protein in different molecular subtypes: normal breast $(n=9)$, luminal A $(n=39)$, luminal B $(n=18)$, Her2 ${ }^{+}(n=19)$, and TNBC $(n=19)$. b Mean Gas6 mRNA expression across distinct molecular subtypes relative to non-cancer and normal-like breast tissues, using METABRIC dataset (non-cancerous, $n=6$; normal-like cancers, $n=140$; luminal A, $n=679$; luminal $\mathrm{B}, n=461$; Her2, $n=219$; basal-like, $n=198$; claudin low, $n=199$ ). c Mean Gas6 mRNA expression across distinct molecular subtypes relative to normallike breast tissues, using TCGA data set (normal-like cancers, $n=8$; luminal $\mathrm{A}, n=212$; luminal $\mathrm{B}, n=119$; Her2, $n=55$; basal-like, $n=87$ ). Middle line is median, box borders are 25th to 75th percentiles and bars are minimum and maximum values. Significant $p$ values are depicted in graphs b-c from one-way ANOVA followed by post hoc Dunnett's multiple comparison test. ns not significant.

Gas6 expression weakly correlates with Axl in invasive breast cancer

Numerous studies in breast cancer models have shown tumorpromoting functions of Gas6 through interactions of the Axl receptor ${ }^{4,6,24}$. In contrast, a recent study using a mouse model of Her2 ${ }^{+}$breast cancer (MMTV-Neu) showed that Axl-mediated metastasis was ligand independent ${ }^{25}$. Therefore, we next asked whether there was an association between Gas6 and Axl protein expression in clinical samples. To address this question, consecutive sections from the same patients were stained with antibodies to either Gas6 or Axl, as shown in Fig. 3a. Antibody specificity was validated by staining $\mathrm{H} 1299$ cells (positive control) or Jurkat cells (negative control) (Supplementary Fig. 4). As with Gas6 scoring (Fig. 1a), Axl expression was scored based on intensity and number of cells stained positive as previously described $^{26}$. Immunohistochemical analysis of normal breast showed complete absence of Axl staining, consistent with previous reports ${ }^{25}$, whereas nearly all ductal epithelium expressed moderate-high levels of Gas6. Axl protein slightly increased in DCIS (primarily tumor epithelial cells), with the most intense expression in IDC (Fig. 3a). Conversely, Gas6 protein expression trended downwards as lesions progressed to IDC (Figs 1c, 3), and there was a weak significant correlation between Gas6 and Axl protein scores ( $r=0.27$, Pearson's correlation, $p=0.0004$ ) (Fig. 3b). Although a subset of cells appeared to co-express Gas6 and Axl, the majority of the Gas6 ${ }^{+}$cells were Axl-negative (Fig. 3a). Next, we performed correlation tests using METABRIC and TCGA data sets. The correlation was significantly moderate between Gas6 mRNA and Axl mRNA when all patients were included without classification. We then performed the test on PAM50-classified groups; in METABRIC data set, the correlation between Gas6 mRNA and Axl mRNA was not significant in the non-cancerous group, yet it was significantly weak in normal-like patients, and moderate in Luminal A, Luminal B, Her2 ${ }^{+}$, basal-like, and Claudin low patients. In TCGA data set, the correlation between Gas6 mRNA and Axl mRNA was not significant in normal-like patients, but it was significantly moderate in Luminal A, Luminal B, Her2 ${ }^{+}$ and basal-like patients (Fig. 3c). These results suggest that epithelial Gas6 in normal breast and potentially breast cancer may have functions both dependent and independent of Axl.

High Gas6 expression is associated with improved overall and RFS Given the higher levels of Gas6 in normal breast and DCIS, and reduction of Gas6 in invasive breast cancer, most prominently in several subtypes known to be aggressive, we asked whether Gas6 was associated with patient survival or prognosis. There was a significant improvement in overall survival in Gas6 high patients in the METABRIC data set in all patients/all treatments $(n=1904, p=$ 0.0004) (Fig. 4a). When stratified by subtype, there was a significant increase in overall survival in Gas6 high luminal A 
a
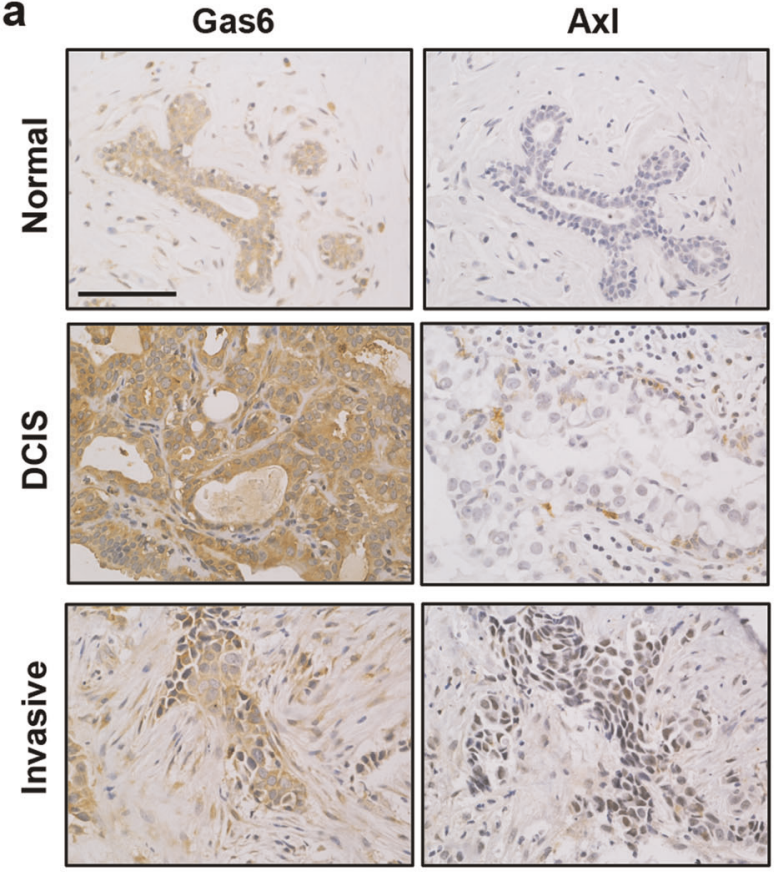

b
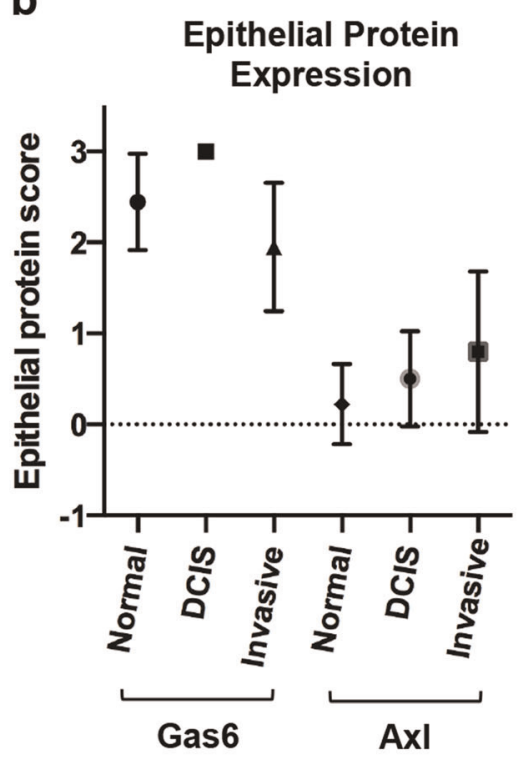

C

Pearson's coefficients for Gas6 and AxI mRNA in different breast cancer subtypes.

\begin{tabular}{|c|c|c|c|c|c|c|c|c|}
\hline METABRIC & $\begin{array}{c}\text { All } \\
\text { patients }\end{array}$ & $\begin{array}{c}\text { Non } \\
\text { cancerous }\end{array}$ & Normal-like & Luminal A & Luminal B & Her2 & Basal-like & Claudin low \\
\hline $\begin{array}{c}\text { Pearson's } \\
\text { coefficient }\end{array}$ & 0.52 & 0.32 & 0.23 & 0.47 & 0.50 & 0.53 & 0.49 & 0.42 \\
\hline p value & $6.80 \mathrm{e}-13$ & 0.53 & 0.005 & $00+\mathrm{e} 00$ & $3.71 \mathrm{e}-31$ & $1.98 \mathrm{e}-17$ & $1.83 \mathrm{e}-13$ & $6.46 \mathrm{e}-10$ \\
\hline TCGA & All patients & Normal-like & Luminal A & Luminal B & Her2 & Basal-like \\
\hline $\begin{array}{c}\text { Pearson's } \\
\text { coefficient }\end{array}$ & 0.53 & 0.62 & 0.52 & 0.51 & 0.57 & 0.43 \\
\hline p value & 0.0001 & 0.09 & $1.28 \mathrm{e}-16$ & $1.62 \mathrm{e}-09$ & $4.91 \mathrm{e}-06$ & $3.13 \mathrm{e}-05$ \\
\hline
\end{tabular}

Pearson's coefficient range: Weak: 0.1-0.3, Moderate: $0.3-0.7$ and Strong 0.7-1

Fig. 3 Differential expression of Gas6 and AxI. a Images depict immunohistochemical staining using antibodies to Gas6 or Axl in normal $(n=9)$, DCIS $(n=12)$ and invasive ductal carcinoma $(n=100)$ from the same patients. Scale bar $=100 \mu \mathrm{m}$. b Graph shows quantitation of Gas6 or Axl staining represented by score (0-3) in normal, DCIS and invasive breast tissues. Error bars are SEM. c Tables represent Pearson's coefficients, and the corresponding $p$ value, between Gas6 mRNA, and AxI mRNA in METABRIC and TCGA data sets, using either all patients data or PAM50-classfied subtypes.

patients as compared with Gas6 low ( $n=679, p=0.003)$ (Fig. 4a), whereas all other subtypes showed no significant changes in overall survival (Supplementary Fig. 5a). In support, tumors with low Gas6 expression are larger in size, have higher cellularity, higher histological grade, and an increased Nottingham Prognostic Index (Table 2), all of which are prognostic markers for patient response to neoadjuvant chemotherapy ${ }^{27}$.

Next, we examined RFS in patients that received treatment, either chemical or hormonal, using the Kaplan-Meier plotter database ${ }^{28}$. RFS was superior in patients with tumors expressing higher levels of Gas6 mRNA, regardless of whether these patients received any treatment $\left(n=3951, p=1.9 \times 10^{-11}\right)$ (Fig. 4b). Gas6 high patients with luminal A breast cancer showed significantly improved RFS over Gas6 low patients $\left(n=1933, p=1.8 \times 10^{-6}\right)$ (Fig. 4b), whereas all other subtypes showed only moderate improvement to no change in RFS (Supplementary Fig. 5b). RFS was not significantly changed when patients that received treatment were stratified by grade (Supplementary Fig. 5b). Taken together, these results suggest that high Gas6 mRNA is associated with increased patient survival and is likely not predictive of poor outcome.

\section{DISCUSSION}

There has been enormous interest in targeting the TAMR family as a therapeutic strategy for metastatic breast cancer, and a number of tyrosine kinase inhibitors to Axl and Mer are currently in clinical trials ${ }^{29}$. Gas6 has primarily been shown to be a macrophagederived factor that activates Axl in mouse models of breast cancer, and Warfarin, an anticoagulant that blocks the interaction between Gas6 and Axl, has recently been proposed as a cancer prevention strategy ${ }^{30,31}$. Despite the known tumor-promoting effects of macrophage Gas6 in vitro and in mouse models, little is known about the relevance and function of tumor-derived Gas6 in human breast cancer. The goals of this study were to identify Gas6 expression patterns in early and late stages of breast cancer and to determine whether tumor-derived Gas6 is associated with breast cancer progression.

We previously showed that Gas6 protein expression was high in mouse p53-null pre-invasive lesions but declined in established tumors $^{18,32}$, suggesting that Gas 6 has a major role in the transition from premalignancy to invasive cancer. Moreover, we showed that macrophage Gas6 induced a malignant phenotype in vitro by 
a METABRIC
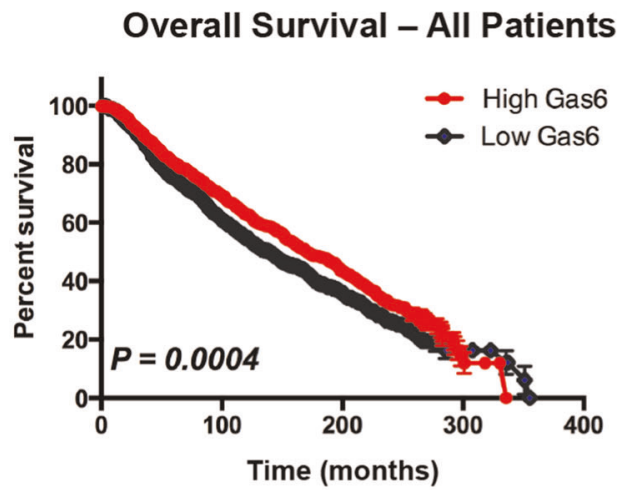

b $\mathrm{KM}$ plotter

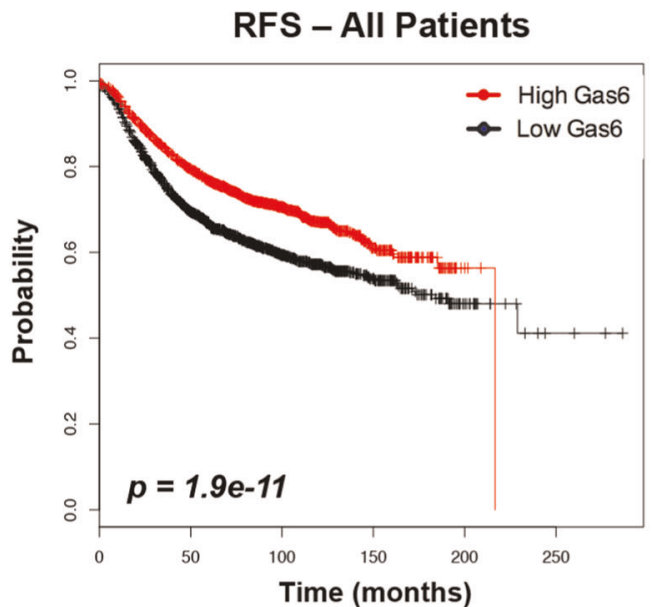

\section{Overall Survival - Luminal A}
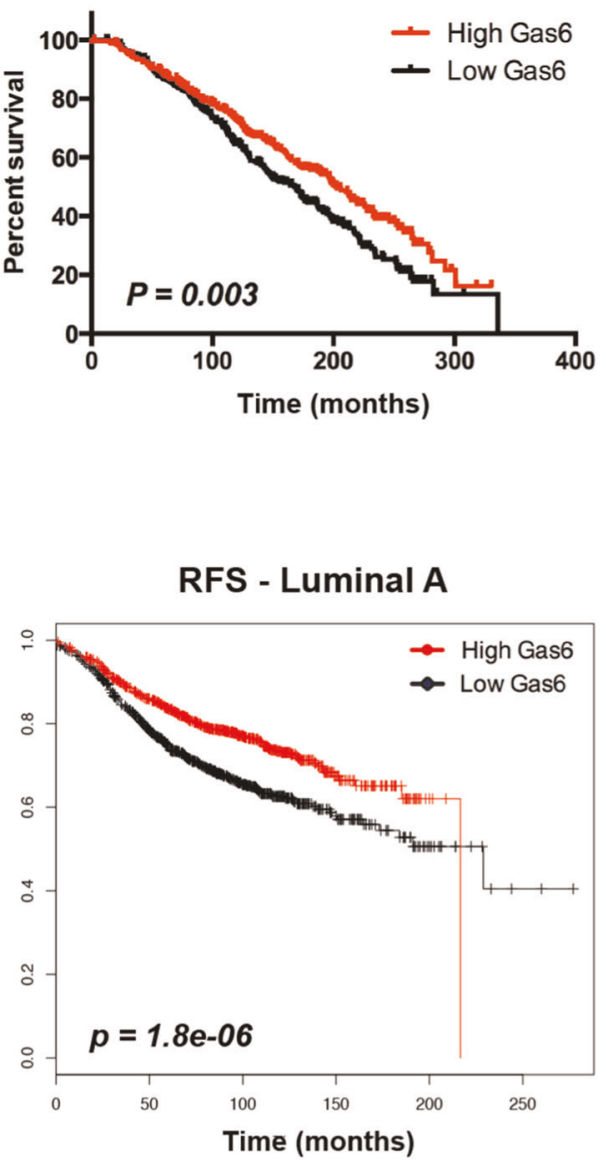

Fig. 4 Higher Gas6 mRNA predicts better survival in breast cancer. a Kaplan-Meier plots generated from METABRIC show overall survival in all patients $(n=1904, p=0.0004)$ (left) and in luminal A patients ( $n=679, p=0.003)$ (right), with high and low levels of Gas6 mRNA. b Kaplan-Meier plots from KMplotter representing relapse-free survival (RFS) of all patients $\left(n=3951, p=1.9 \times 10^{-11}\right)$ (left), and in luminal A patients $\left(n=1933, p=1.8 \times 10^{-6}\right)$ (right) with high and low levels of Gas6 mRNA. Log-rank, Chi-squared test for statistical analysis.

activating Axl on pre-invasive cells, however in vivo, once tumors were established, Gas6 was not required for further progression ${ }^{18}$. These results led us to directly address whether Gas6 protein expression decreased during human breast cancer progression. Here, we showed that Gas6 is highly expressed in the ductal epithelial cells of normal breast with faint stromal expression (Fig. 1), which is consistent with our previous studies of the normal murine mammary gland ${ }^{19}$. Epithelial-derived Gas 6 protein expression was significantly increased in DCIS, but declined in invasive tumors (Fig. 1), supporting our published studies in mouse models ${ }^{18}$.

Early studies using a small cohort of patients showed that Gas6 mRNA is expressed in human breast cancer and correlates with progesterone receptor and favorable clinical parameters ${ }^{33}$. In support of these findings, we found that Gas6 showed the lowest protein expression in TNBC when stratified by subtype (Fig. 2a). Analysis of the METABRIC and TCGA data sets showed the highest Gas6 mRNA levels in luminal A breast cancer and then a decline towards luminal $\mathrm{B}, \mathrm{Her}^{+}$, and basal-like (Fig. 2b, c). luminal A breast cancer typically has the best prognosis among other subtypes, considering the response to therapy and the slow growth of cancer cells attributed to the low levels of Ki67 expression. Luminal B and Her2 ${ }^{+}$subtypes generally have lower overall survival owing to their fast growth and tendency to relapse after removal. Likewise, basal-like breast cancer is associated with poor overall survival, partly due to increased metastatic potential and a lack of targeted therapeutic strategies $22,34,35$, although a proportion of these patients are associated with good prognosis ${ }^{36}$. These observations led us to ask whether Gas6 expression in human breast cancers correlates with overall survival. Analysis of the METABRIC data set that includes all patients regardless of treatment revealed a significant increase in overall survival in patients with high Gas6 mRNA (Fig. 4a). Using the Kaplan-Meier plotter database (KMplotter) ${ }^{28}$, we found that high Gas6 mRNA correlated to improved RFS in breast cancer patients, particularly in patients with luminal A breast cancer, when stratified with those who had received therapy (Fig. 4b). These findings suggest that Gas6 protein or mRNA expression in human breast tumors may not be a negative prognostic factor.

Similar to earlier studies associating Gas6 expression with favorable clinical outcome in breast cancer, Gas6 was shown to inhibit intestinal tumorigenesis in a mouse model of intestinal cancer, and Gas6 protein expression in human colorectal cancers positively correlated with prognosis ${ }^{37}$. However, numerous other reports suggest that Gas6 is tumor promoting in various cancers including colon, thyroid, lung, and ovarian (reviewed in ref. ${ }^{38}$ ). In breast cancer, studies have largely focused on Axl, and targeting Axl or Gas6-Axl has been proposed. Axl has been correlated with 
AM Ibrahim et al.

Table 2. Clinical attributes of patients with low and high levels of Gas6 in METABRIC data set.

\begin{tabular}{llll}
\hline Clinical attribute & Low Gas6 mRNA & High Gas6 mRNA & $p$ value \\
\hline Tumor Size $(n=1854)$ & $26.99 \pm 0.524$ & $25.57 \pm 0.466$ \\
Neoplasm histologic grade $(n=1780)$ & $2.525 \pm 0.022$ & $2.32 \pm 0.022$ & $0.043(*)$ \\
Nottingham prognostic index $(n=1850)$ & $4.195 \pm 0.0367$ & $3.891 \pm 0.0377$ & $0.0001(* * * *)$ \\
Cellularity $(n=1139)$ & $9.663 \pm 0.7805$ & $9.150 \pm 0.7762$ & $0.0001(* * * *)$ \\
\hline
\end{tabular}

poor prognosis in breast cancer patients and staining of clinical samples showed that $\mathrm{Axl}$ is overexpressed in a small percentage of TNBC and $\mathrm{Her}^{+}$patients $^{13,25}$. In this study, tumor-derived Gas6 protein did not strongly correlate to Axl expression (Fig. 3). In support, numerous studies have shown Gas6-independent activation of Axl in cancer. Goyette et al. showed that genetic ablation of AxI in MMTV-Neu tumors results in decreased tumor formation and metastasis, although Gas6 deletion had no effect on mammary tumorigenesis ${ }^{25}$. Protein $\mathrm{S}$, which is highly homologous to Gas6, was shown to regulate Axl and drive tumorigenesis in a mouse model of oral squamous cell carcinoma ${ }^{39}$. Protein $S$ was also shown to activate Axl in glioblastoma, contributing to growth of aggressive tumors ${ }^{40}$. Additional Gas6-independent mechanisms involving interactions of Axl with other RTKs including EGFR ${ }^{41}$, $\mathrm{MET}^{42}$, and VEGFR2 ${ }^{43}$ have also been described. Thus, it is plausible that Axl-mediated breast cancer progression may not require Gas6. It is also possible that low levels of Gas6 may be required to sustain Axl activation in tumor cells. Additional functional studies are required to fully understand these mechanisms.

In addition to reports of ligand-independent Axl-induced tumor progression, numerous studies suggest that targeting Gas6 or Gas6/Axl in breast cancer is beneficial. In ovarian cancer cell lines derived from mesenchymal (Mes) or epithelial (Epi-A) mouse models, Gas6-stimulated AxI-RTK crosstalk in Mes cells, whereas Gas6-induced Axl activation did not involve other RTKs in Epi-A cells $^{44}$. Leukocyte-derived Gas6 was shown to mediate tumor growth in a syngeneic TNBC mouse model (4T1) as well as in other cancer cell models ${ }^{12}$. Kariolis et al. ${ }^{45}$ described an Axl "decoy receptor" that sequesters Gas6 and was shown to inhibit metastasis in ovarian cancer xenografts and a syngeneic TNBC mouse model (4T1). Of note, the decoy receptor not only blocks the Gas6-Axl interaction, but also other Gas6 receptors, including Mer. In another study, dual inhibition of Gas6 and Mer decreased tumor-associated macrophages, increased $\mathrm{CD} 4^{+} \mathrm{T}$ cells and reduced tumor formation in lung cancer cells in vivo ${ }^{46}$. Gas6 has also been shown to enhance $\mathrm{T}$ regulatory cell (Treg) suppressor activity by binding Axl on Tregs $^{47}$. In the present study, our data showed that stromal Gas6 expression did not significantly change during breast cancer progression (Supplemental Fig. 1), however it remains unclear as to whether macrophage-derived Gas6, or Gas6 from other stromal cells, has tumor-promoting activities. Despite the observed decreased in tumor-derived Gas6 in advanced breast cancers, targeting stromal Gas6 and its receptors remains a promising therapeutic approach and may be valuable when combined with immunotherapies ${ }^{40,48}$. Further studies are required to understand the biological function of Gas6 in human breast cancer.

\section{METHODS}

Patient samples and ethics

A TMA (BC081116C) containing samples of 100 patient tissues of various breast cancer subtypes and nine adjacent normal breast (referred to as "normal" throughout the manuscript) were purchased from US Biomax (Biomax website states that tissues were collected upon the donors written consent under HIPPA approved protocols). TMAs containing 12 patient samples with DCIS, and 44 IDC patient samples with the corresponding IDC-associated DCIS, were kindly obtained from Dr. Fariba Behbod
(University of Kansas Medical Center, KS, USA). All patients gave written informed consent for participation in this University of Kansas Medical Center Institutional Review Board-approved study allowing collection of additional biopsy and or surgical tissue for research. Histopathological characteristics are listed in Table 1.

\section{Immunostaining}

Paraffin-embedded TMAs sections $(5 \mu \mathrm{m})$ and paraffin-embedded cell pellets (NCl-H226, HT29, H1299 and Jurkat cells (Cell signaling) were deparaffinized, rehydrated, and antigen retrieval was performed in the microwave using $10 \mathrm{mM}$ sodium citrate for 20 mins. For immunohistochemistry (IHC), endogenous peroxidases were quenched with $3 \% \mathrm{H}_{2} \mathrm{O}_{2}$ in methanol, blocked with $5 \%$ bovine serum albumin (BSA) in PBS containing $0.05 \%$ Tween and incubated with a Gas6 antibody (R\&D \#AF885, 1:50 dilution) and Axl antibody (Cell Signaling Technology \#8661 S, 1:3000 dilution) overnight at $4{ }^{\circ} \mathrm{C}$. The next day, slides were washed with PBS and incubated with a biotinylated antibody (1:500) (Vector Laboratories) for $30 \mathrm{~min}$. Slides were washed with PBS, incubated for $10 \mathrm{~min}$ with the VECTASTAIN Elite ABC-HRP reagent, R.T.U (Vector Laboratories), and developed using a DAB peroxidase substrate kit (Vector Laboratories). Sections were then counterstained with hematoxylin, dehydrated and mounted with permount (Fisher Scientific). Images were acquired using a Nikon Eclipse microscope (Nikon Instruments). For immunofluorescence, sections were blocked with 5\% BSA in PBS containing $0.05 \%$ Tween for $1 \mathrm{~h}$ at RT and incubated overnight at $4{ }^{\circ} \mathrm{C}$, with Gas6 antibody (1:50 dilution) and Axl antibody (1:3000 dilution). The next day, slides were washed with PBST, and incubated in dark with fluorescent 2ry antibody (1:500, Alexaflour 488, Thermo Scientific) for $1 \mathrm{~h}$. Slides were then washed with PBST and nuclei were counterstained (Vector laboratories). Fluorescent images were captured with Nikon confocal microscope (Nikon instruments). For IHC antibody scoring, tissues were examined using $\times 20$ and $\times 40$ objectives. An arbitrary number was given on a scale of $0-3$ by visual examination, based on the intensity and the number of stained cells, taking any field to field variation into account, where 0 was assigned for negative staining, 1 for low, 2 for moderate, and 3 for strong staining ${ }^{26}$. A separate score was assigned for either epithelial or stromal staining, and all samples were scored blindly by two independent individuals.

\section{Online data and statistical analyses}

Epithelial Gas6 score in TMAs sections was statistically analyzed using Chisquared test for pair-wise comparison. Data were considered statistically significant if $P$ values $\leq 0.01$. A Pearson's correlation coefficient was calculated to assess the relationship between the scores of Gas6 and AxI protein expression using GraphPad Prism8. Gas6 mRNA expression in normal and tumor tissues was gathered from GEPIA, an online platform with RNA-seq data from TCGA and GTEx databases ${ }^{20}$. Gas6 transcripts per million from both normal and tumor tissue were plotted using one-way analysis of variance (ANOVA) differential method and a $q$ value cutoff of 0.01 . METABRIC and TCGA data were accessed through cBioportal and was further categorized using the Pam50 classification ${ }^{22}$. Patient Gas6 mRNA levels were matched with the appropriate sample-ID. With median Gas6 expression as the cutoff value, GraphPad Prism software was used to calculate statistical differences of mean Gas6 expression between normal and breast cancer subtypes using one-way ANOVA with post hoc Dunnett's multiple comparison test. Correlating Gas6 mRNA and Axl mRNA in METABRIC and TCGA datasets was performed using the Pearson's correlation module in GraphPad Prism, and using Gas6 and AxI mRNA values from PAM50-classified patients subtypes. Survival curves were generated using two data sets: METABRIC data set was mined and the overall survival status of patients with different subtypes and the corresponding Gas6 mRNA level per patient were downloaded and 
grouped as high and low, based on Gas6 mRNA expression level and using the median expression as a cutoff. Survival graphs were then plotted using survival module in GraphPad Prism8. The second dataset was Kaplan-Meier Plotter (KMplotter), an online platform combining gene microarray data and patient survival rates from Gene Expression Omnibus (Affymetrix HGU133A and HGU133+2 microarrays) ${ }^{28}$. Patients were divided using an auto selection feature based on median and quartile expression levels of Gas6 (valid Affy ID: 1598_g_at) and quality controlled for redundant samples and biased assays. Median survival was reported in months and compared for significance with a hazard ratio and $p$ value generated on the graph. A $p$ value of $<0.05$ was considered statistically significant (Log-rank, Chi-squared test). Overall survival and RFS were tested without further criteria filtering. RFS for subtypes were restricted to treated patients cohort, and the subtypes selection was an intrinsic grouping of patients based on their gene expression.

\section{Reporting summary}

Further information on research design is available in the Nature Research Reporting Summary linked to this article.

\section{DATA AVAILABILITY}

Clinical and histopathological data for the TMAs acquired from the US Biomax can be accessed from the US biomax website (https://www.biomax.us/tissue-arrays/Breast/ BC081116c) (Table 1). GEPIA data set analyzed in this study can be accessed via the website (http://gepia.cancer-pku.cn/index.html) (Supplementary Fig. 2). The METABRIC and the TCGA breast cancer data sets analyzed during this study can be accessed from the cBioPortal for Cancer Genomics repository (https://www.cbioportal.org/) (Figs 2b, c, 4a, and Table 2). KM plotter data set analyzed in this study can be accessed from KM plotter website (http://kmplot.com/analysis/index.php?p=service) (Fig. 4b and Supplementary Fig. 5).

Received: 2 October 2019; Accepted: 13 March 2020; Published online: 24 April 2020

\section{REFERENCES}

1. Geng, K. et al. Requirement of gamma-carboxyglutamic acid modification and phosphatidylserine binding for the activation of Tyro3, Axl, and mertk receptors by growth arrest-specific 6. Front. Immunol. 8, 1521 (2017).

2. Tsou, W. I. et al. Receptor tyrosine kinases, TYRO3, AXL, and MER, demonstrate distinct patterns and complex regulation of ligand-induced activation. J. Biol. Chem. 289, 25750-25763 (2014).

3. Demarchi, F., Verardo, R., Varnum, B., Brancolini, C. \& Schneider, C. Gas6 antiapoptotic signaling requires NF-kappa B activation. J. Biol. Chem. 276, 31738-31744 (2001).

4. Zweemer, A. J. M. et al. Apoptotic bodies elicit Gas6-mediated migration of AXLexpressing tumor cells. Mol. Cancer Res. 15, 1656-1666 (2017).

5. Tjwa, M. et al. Gas 6 promotes inflammation by enhancing interactions between endothelial cells, platelets, and leukocytes. Blood 111, 4096-4105 (2008).

6. Han, J. et al. Gas6/AxI mediates tumor cell apoptosis, migration and invasion and predicts the clinical outcome of osteosarcoma patients. Biochem. Biophys. Res. Commun. 435, 493-500 (2013).

7. Lee, H. J., Jeng, Y. M., Chen, Y. L., Chung, L. \& Yuan, R. H. Gas6/Axl pathway promotes tumor invasion through the transcriptional activation of Slug in hepatocellular carcinoma. Carcinogenesis 35, 769-775 (2014).

8. Rankin, E. B. et al. Direct regulation of GAS6/AXL signaling by HIF promotes renal metastasis through SRC and MET. Proc. Natl Acad. Sci. USA 111, 13373-13378 (2014).

9. Wimmel, A., Glitz, D., Kraus, A., Roeder, J. \& Schuermann, M. Axl receptor tyrosine kinase expression in human lung cancer cell lines correlates with cellular adhesion. Eur. J. Cancer 37, 2264-2274 (2001).

10. Leconet, $W$. et al. Therapeutic activity of anti-AXL antibody against triple-negative breast cancer patient-derived xenografts and metastasis. Clin. Cancer Res. 23 2806-2816 (2017).

11. Verma, A., Warner, S. L., Vankayalapati, H., Bearss, D. J. \& Sharma, S. Targeting Axl and Mer kinases in cancer. Mol. Cancer Ther. 10, 1763 (2011).

12. Loges, S. et al. Malignant cells fuel tumor growth by educating infiltrating leukocytes to produce the mitogen Gas6. Blood 115, 2264 (2010).

13. Gjerdrum, C. et al. Axl is an essential epithelial-to-mesenchymal transitioninduced regulator of breast cancer metastasis and patient survival. Proc. Nat Acad. Sci. USA 107, 1124-1129 (2010).
14. Li, Y. et al. Axl as a potential therapeutic target in cancer: role of $\mathrm{Axl}$ in tumor growth, metastasis and angiogenesis. Oncogene 28, 3442 (2009).

15. D'Errico, G. et al. Tumor-associated macrophage-secreted $14-3-3 \zeta$ signals via AXL to promote pancreatic cancer chemoresistance. Oncogene 38, 5469-5485 (2019).

16. Antony, J. \& Huang, R. Y.-J. AXL-driven EMT state as a targetable conduit in cancer. Cancer Res. 77, 3725 (2017).

17. Vouri, M. \& Hafizi, S. TAM receptor tyrosine kinases in cancer drug resistance. Cancer Res. 77, 2775-2778 (2017).

18. Gomes, A. M. et al. Stromal Gas6 promotes the progression of premalignant mammary cells. Oncogene 38, 2437-2450 (2019).

19. Mills, K. L. et al. Gas6 is dispensable for pubertal mammary gland development. PLOS ONE 13, e0208550 (2018).

20. Tang, Z. et al. GEPIA: a web server for cancer and normal gene expression profiling and interactive analyses. Nucleic Acids Res. 45, W98-W102 (2017).

21. Cerami, E. et al. The cBio cancer genomics portal: an open platform for exploring multidimensional cancer genomics data. Cancer Discov. 2, 401 (2012).

22. Curtis, C. et al. The genomic and transcriptomic architecture of 2,000 breast tumours reveals novel subgroups. Nature 486, 346-352 (2012).

23. The Cancer Genome Atlas, N. et al. Comprehensive molecular portraits of human breast tumours. Nature 490, 61 (2012).

24. Ben-Batalla, I. et al. Axl, a prognostic and therapeutic target in acute myeloid leukemia mediates paracrine crosstalk of leukemia cells with bone marrow stroma. Blood 122, 2443 (2013).

25. Goyette, M. A. et al. The receptor tyrosine kinase AXL is required at multiple steps of the metastatic cascade during HER2-positive breast cancer progression. Cell Rep. 23, 1476-1490 (2018).

26. Ibrahim, A. M. et al. Fibulin-2 is required for basement membrane integrity of mammary epithelium. Sci. Rep. 8, 14139 (2018).

27. Rajan, R. et al. Change in tumor cellularity of breast carcinoma after neoadjuvant chemotherapy as a variable in the pathologic assessment of response. Cancer 100, 1365-1373 (2004).

28. Gyorffy, B. et al. An online survival analysis tool to rapidly assess the effect of 22,277 genes on breast cancer prognosis using microarray data of 1809 patients. Breast Cancer Res. Treat. 123, 725-731 (2010).

29. Graham, D. K., DeRyckere, D., Davies, K. D. \& Earp, H. S. The TAM family: phos phatidylserine sensing receptor tyrosine kinases gone awry in cancer. Nat. Rev. Cancer 14, 769-785 (2014).

30. Haaland, G. S., Falk, R. S., Straume, O. \& Lorens, J. B. Association of warfarin use with lower overall cancer incidence among patients older than 50 years. JAMA Intern. Med. 177, 1774-1780 (2017).

31. Kirane, A. et al. Warfarin blocks Gas6-mediated Axl activation required for pancreatic cancer epithelial plasticity and metastasis. Cancer Res. 75, 3699-3705 (2015)

32. Carron, E. C. et al. Macrophages promote the progression of premalignant mammary lesions to invasive cancer. Oncotarget 8, 50731-50746 (2017).

33. Mc Cormack, O. et al. Growth arrest-specific gene 6 expression in human breast cancer. Br. J. Cancer 98, 1141-1146 (2008).

34. Dent, R. et al. Triple-negative breast cancer: clinical features and patterns of recurrence. Clin. Cancer Res. 13, 4429 (2007).

35. Fragomeni, S. M., Sciallis, A. \& Jeruss, J. S. Molecular subtypes and local-regional control of breast cancer. Surg. Oncol. Clin. N. Am. 27, 95-120 (2018).

36. Ungerleider, N. A. et al. Breast cancer survival predicted by TP53 mutation status differs markedly depending on treatment. Breast Cancer Res 20, 115-115 (2018).

37. Akitake-Kawano, R. et al. Inhibitory role of Gas6 in intestinal tumorigenesis. Carcinogenesis 34, 1567-1574 (2013).

38. Wu, G. et al. Molecular insights of Gas6/TAM in cancer development and therapy. Cell Death Dis. 8, e2700 (2017).

39. Abboud-Jarrous, $G$. et al. Protein $S$ drives oral squamous cell carcinoma tumorigenicity through regulation of AXL. Oncotarget 8, 13986-14002 (2017).

40. Sadahiro, $H$. et al. Activation of the receptor tyrosine kinase $A X L$ regulates the immune microenvironment in glioblastoma. Cancer Res. 78, 3002-3013 (2018).

41. Meyer, A. S., Miller, M. A., Gertler, F. B. \& Lauffenburger, D. A. The receptor AXL diversifies EGFR signaling and limits the response to EGFR-targeted inhibitors in triple-negative breast cancer cells. Sci. Signal 6, ra66 (2013).

42. Salian-Mehta, S., Xu, M. \& Wierman, M. E. AXL and MET crosstalk to promote gonadotropin releasing hormone $(\mathrm{GnRH})$ neuronal cell migration and survival. Mol. Cell Endocrinol. 374, 92-100 (2013).

43. Ruan, G. X. \& Kazlauskas, A. Axl is essential for VEGF-A-dependent activation of PI3K/Akt. Embo J. 31, 1692-1703 (2012).

44. Antony, J. et al. The GAS6-AXL signaling network is a mesenchymal (Mes) molecular subtype-specific therapeutic target for ovarian cancer. Sci. Signal $\mathbf{9}$ ra97 (2016).

45. Kariolis, M. S. et al. An engineered Axl 'decoy receptor' effectively silences the Gas6-Axl signaling axis. Nat. Chem. Biol. 10, 977-983 (2014).

46. Novitskiy, S. V. et al. Gas6/MerTK signaling is negatively regulated by NF-kappaB and supports lung carcinogenesis. Oncotarget 10, 7031-7042 (2019). 
47. Zhao, G. J. et al. Growth arrest-specific 6 enhances the suppressive function of $\mathrm{CD} 4(+) \mathrm{CD} 25(+)$ regulatory $\mathrm{T}$ cells mainly through Axl receptor. Mediators Inflamm. 2017, 6848430 (2017).

48. Leconet, W. et al. Preclinical validation of AXL receptor as a target for antibodybased pancreatic cancer immunotherapy. Oncogene 33, 5405-5414 (2014).

\section{ACKNOWLEDGEMENTS}

We thank Ashkan Shahbandi (Tulane School of Medicine) for help with data analysis, and Dr. James Jackson (Tulane School of Medicine) for critical scientific discussion. We acknowledge support from the University of Kansas (KU) Cancer Center's Biospecimen Repository Core Facility staff for helping obtain human specimens. This work was supported by the KU Cancer Center's Cancer Center Support Grant (P30 CA168524), R21CA185460 (F.B.), American Association for Cancer Research (AACR)2014 Breast Cancer Research Foundation-Translational Breast Cancer research (F.B.), NIH R01 CA212518 (H.L.M.), and Susan G. Komen CCR16377665 (H.L.M.).

\section{AUTHOR CONTRIBUTIONS}

A.M.I., Z.G. and A.M.G. (co-first authors): conception and design, collection and assembly of data, data analysis and interpretation, manuscript writing. L.M.: statistical analysis of data. F.B.: collection and assembly of data, interpretation of results. H.L.M.: conception and design, collection and assembly of data, data analysis and interpretation, manuscript writing, financial support.

\section{COMPETING INTERESTS}

The authors declare no competing interests.

\section{ADDITIONAL INFORMATION}

Supplementary information is available for this paper at https://doi.org/10.1038/ s41698-020-0116-z.

Correspondence and requests for materials should be addressed to H.L.M.

Reprints and permission information is available at http://www.nature.com/ reprints

Publisher's note Springer Nature remains neutral with regard to jurisdictional claims in published maps and institutional affiliations.

cc) (i)

Open Access This article is licensed under a Creative Commons Attribution 4.0 International License, which permits use, sharing, adaptation, distribution and reproduction in any medium or format, as long as you give appropriate credit to the original author(s) and the source, provide a link to the Creative Commons license, and indicate if changes were made. The images or other third party material in this article are included in the article's Creative Commons license, unless indicated otherwise in a credit line to the material. If material is not included in the article's Creative Commons license and your intended use is not permitted by statutory regulation or exceeds the permitted use, you will need to obtain permission directly from the copyright holder. To view a copy of this license, visit http://creativecommons. org/licenses/by/4.0/.

(c) The Author(s) 2020 